\title{
Riesgo de Caries en Niños que Ingresan al Programa de Población en Control con Enfoque de Riesgo Odontológico
}

\author{
Dental Caries Risk in Children who Enter the Program \\ Population in Control with Dental Risk Approach
}

Ariel Nenen'; Constanza Courdurier² \& Alexander Arcos $^{3}$

\begin{abstract}
NENEN, A.; COURDURIER, C. \& ARCOS, A. Riesgo de caries en niños que ingresan al programa de Población en Control con Enfoque de Riesgo Odontológico. Int. J. Odontostomat., 13(4):437-441, 2019.

RESUMEN: El objetivo de este estudio fue determinar el riesgo de caries en la población infantil entre 7 y 34 meses, usuarios de un centro de atención primaria de salud en la ciudad de Osorno, ingresados al programa de Población en Control con Enfoque de Riesgo Odontológico (CERO) durante el año 2017. Expresándolo en alto o bajo riesgo. Se realizó un estudio observacional descriptivo, tomando una muestra de 135 pacientes, con edades dentro del rango en estudio, inscritos en el centro de salud e ingresados al programa. Se utilizaron las pautas diseñadas por el Ministerio de Salud e incluidas en el programa CERO, aplicándolas en una sesión, junto al examen clínico para determinar el nivel de riesgo de cada individuo. Posteriormente se sometieron los datos a análisis de estadística descriptiva. El 59 \% de la población entre 7-34 meses tiene un riesgo de caries alto. El grupo con mayor riesgo es el de 12-23 meses con un $70 \%$. Los hombres tienen un $59 \%$ de riesgo alto y las mujeres un $58 \%$. Al analizar en detalle cada variable, el "cepillado antes de dormir" fue el que con mayor frecuencia se encontraba en alto riesgo, con un $60 \%$, seguido del "momento de ingesta de alimentos azucarados" y el "uso de pasta con flúor", con un $56 \%$ y $53 \%$, respectivamente.
\end{abstract}

PALABRAS CLAVE: caries dental, factores de riesgo, salud infantil, salud pública, cuidado dental.

\section{INTRODUCCIÓN}

En Chile, la caries tiene una prevalencia de 17,5 $\%$ a los 2 años (Letelier et al., 2012) alcanzando el 70 $\%$ a los 6 años (Ministerio de Salud, 2007). La población de nivel socioeconómico bajo y medio son las más afectadas en comparación con el segmento de mayor ingreso y al considerar la condición urbano-rural, se concluye que la probabilidad de que un niño de 6 años se encuentre libre de caries es el doble en zonas urbanas que en zonas rurales (Ministerio de Salud, 2007). Estos datos llevan a concluir que la caries dental está influenciada por determinantes sociales y por la exposición a factores de riesgo (Arteaga et al., 2009). El riesgo cariogénico, definido como la probabilidad de incidencia de caries en un período de tiempo determinado, que implica además los cambios en el tamaño y/o actividad de lesiones ya presentes, es fundamental para la toma de decisiones, manejo clínico y planificación (Ministerio de Salud,
2009). Cuenta con variados instrumentos para su medición, pero ninguno es confiable, ni tampoco son comparables entre sí (Hänsel Petersson et al., 2002; Gómez, 2008; Giacaman et al., 2013). Por lo tanto, los instrumentos y los estudios existentes representan un problema al momento de servir como base para aventurar conclusiones y para la creación de políticas públicas de salud. El programa de Población en Control con Enfoque de Riesgo Odontológico (CERO) cuenta con pautas desarrolladas por el Ministerio de Salud (MINSAL) para la determinación del riesgo en la población atendida en los servicios de atención primaria de salud (Ministerio de Salud, 2017). Haciendo uso de estas pautas, el objetivo de este estudio fue determinar el riesgo cariogénico de la población infantil entre 7 y 34 meses, usuarios de un centro de atención primaria de salud en la ciudad de Osorno, el año 2017.

\footnotetext{
${ }^{1}$ Instituto de Odontoestomatología, Facultad de Medicina, Universidad Austral de Chile, Valdivia, Chile.

${ }^{2}$ Escuela de Odontología, Facultad de Medicina, Universidad Austral de Chile, Valdivia, Chile.

${ }^{3}$ CESFAM Quinto Centenario, Osorno, Chile.
} 


\section{MATERIAL Y MÉTODO}

Diseño de estudio: Se diseñó un estudio observacional descriptivo. Se llevó a cabo en un Centro de Salud Familiar (CESFAM), perteneciente a la Ilustre Municipalidad de Osorno durante los meses de abril a junio del año 2017. El estudio fue evaluado y aprobado por el Subcomité de Bioética de la Facultad de Medicina de la Universidad Austral de Chile.

Población y tamaño de muestra: Los pacientes fueron identificados desde los registros de inscritos del CESFAM. Se incluyeron en el estudio a aquellos pacientes mayores de 6 meses y menores de 3 años, que ingresaron al programa CERO y cuyos cuidadores accedieron telefónicamente a asistir a la cita programada. Se excluyeron a aquellos pacientes fuera del rango etario de la población objetivo, a quienes no fue posible contactar vía telefónica y quienes por razones de enfermedad, trabajo u horarios no pudieron asistir.

El tamaño muestral, se calculó en base a los inscritos en los registros del CESFAM (200 pacientes), realizando una aleatorización simple, con un nivel de confianza del $95 \%$, asumiendo una pérdida del $25 \%$. Quedando finalmente un tamaño muestral de 135 pacientes.

Materiales y métodos: Los pacientes fueron citados en grupos de 5, se explicaron los objetivos y el tipo de estudio a realizar. Posteriormente, por parte de uno de los investigadores se entregaron las indicaciones generales al grupo y de manera individual a quien lo requiriese, para el llenado de las pautas del programa CERO por parte del padre, madre y/o cuidador(a). Posteriormente se realizó el examen clínico a cada uno de los menores (cuando correspondía, según edad) y se finalizó la sesión con un taller educativo a los padres y/o cuidadores.

Pautas Programa CERO: Las pautas de riesgo desarrolladas para el programa CERO se consideran una herramienta de tamizaje o screening. Término definido como: "el proceso de identificar a aquellas personas que tienen un riesgo suficientemente alto de tener una condición específica, que amerita una evaluación mayor o una acción directa" (Ministerio de Salud, 2017). Esta consta de 6 ámbitos o variables a evaluar en el menor: 1) Anamnesis, 2) Condición clínica, 3) Dieta, 4) Higiene, 5) Acceso a fluoruros y 6) Motivación:

1) Anamnesis: Se realizan dos preguntas: a) ¿el niño(a) presenta una condición que disminuya su flujo salival (enfermedades, fármacos, etc.)? y b) ¿el niño(a) presenta situación de discapacidad?

2) Condición clínica: Consta de dos preguntas: a) ¿cuál es la historia de caries del niño(a)? y b) ¿cuál es el estado de las encías del niño(a)? En esta variable se realiza examen clínico y construcción de índice ceod. A partir del año de edad.

3) Dieta: Se realizan tres preguntas: a) ¿Cuántas veces al día el niño(a) ingiere alimentos y/o líquidos azucarados?, b) ¿en qué momentos el niño(a) realiza la ingesta de alimentos y/o líquidos azucarados? y c) si el niño(a) toma mamadera, ¿Cuántas veces se queda dormido con ella?

4) Higiene: Se responden tres preguntas: a) ¿Cuántas veces al día el niño(a) se lava los dientes?, b) ¿el niño(a), se lava los dientes antes de ir a dormir? y c) Los padres y/o cuidadores, ¿ayudan al niño(a) a lavarse los dientes?

5) Fluoruros: Solo se responde a ¿utiliza el niño(a) pasta con flúor? Se deben considerar pastas fluoruradas con más de 1000 ppm y se debe responder este ámbito después del año de edad.

6) Motivación de los padres/cuidadores: El dentista examinador (investigador) debe responder a: ¿Cuál cree que es la motivación de los padres en el cuidado oral del niño(a)? Se refiera a la percepción y debe considerar el cuidado integral del menor (higiene general, cuidado personal, trato, etc.)

Las pautas son específicas para cada segmento etario, es decir, existe una pauta para menores entre 6 meses y 11 meses, otra para menores entre 12 meses y 23 meses y para niños entre 24 y 35 meses.

Categorización del riesgo: Las respuestas a cada pregunta de las variables son de naturaleza dicotómica, por lo tanto, una califica para bajo riesgo y la otra para alto riesgo. El riesgo finalmente se obtiene mediante el siguiente criterio:

a) Alto riesgo: Si en las áreas de anamnesis o condición clínica, una de las respuestas es de alto riesgo. Automáticamente se considera en alto riesgo, independiente de los demás resultados. O si en alguno de las áreas de dieta, higiene, fluoruros o motivación de los padres/cuidadores hay por lo menos dos áreas de alto riesgo (se considera de alto riesgo una variable 
cuando más de la mitad de la respuesta sea de alto riesgo), aun cuando las variables de anamnesis y condición clínica sean de bajo riesgo.

b) Bajo riesgo: Si las áreas de anamnesis y condición clínica son de bajo riesgo y al menos tres de las otras áreas son de bajo riesgo, se considera de bajo riesgo.

\section{RESULTADOS}

La población en estudio quedó constituida por 135 pacientes, con edades entre los 7 y 34 meses, con una edad promedio de 18 meses. Un $53 \%$ de sexo masculino y $47 \%$ de sexo femenino. Los grupos por rango etario se distribuyeron en un $34 \%$ para la

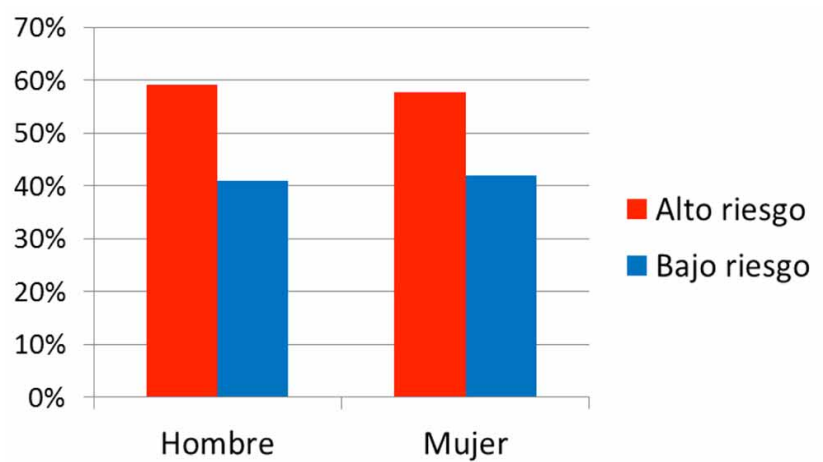

Fig. 1. Riesgo cariogénico por sexo: $59 \%$ del total de hombres y $58 \%$ del total de mujeres presentan riesgo alto. población entre $7-11$ meses, $33 \%$ de $12-23$ meses y $33 \%$ de 24-34 meses (Tabla I).

El $59 \%$ del total de hombres presenta riesgo cariogénico alto, mientras que en el caso de las mujeres un $58 \%$ es de alto riesgo (Fig. 1).

El $50 \%$ de la población de entre 7-11 meses tiene riesgo alto. El mayor porcentaje lo exhibe la población entre los $12-23$ meses, con un $70 \%$ de riesgo alto y en el segmento de $24-34$ meses el $58 \%$ presenta alto riesgo. Finalmente, el $59 \%$ de la población infantil entre 7 y 34 meses presenta un riesgo cariogénico alto (Fig. 2).

El análisis en detalle de las variables evaluadas en las pautas CERO muestran que en el grupo de 7-11 meses, el factor que se pesquisó con mayor frecuencia en alto riesgo fue el "cepillado antes de dormir" con un $65 \%$, seguido por "mamadera antes de dormir" con un 50 \% y luego "frecuencia de cepillado" con un $43 \%$.

En el grupo 12-23 meses, los factores que se encontraron en alto riesgo fueron "uso de pasta con flúor", con un $89 \%$, seguido de "cepillado antes de dormir" y "momento de ingesta de azucares", ambos con un $70 \%$. Además este grupo fue el que presentó mayor cantidad de factores en alto riesgo.

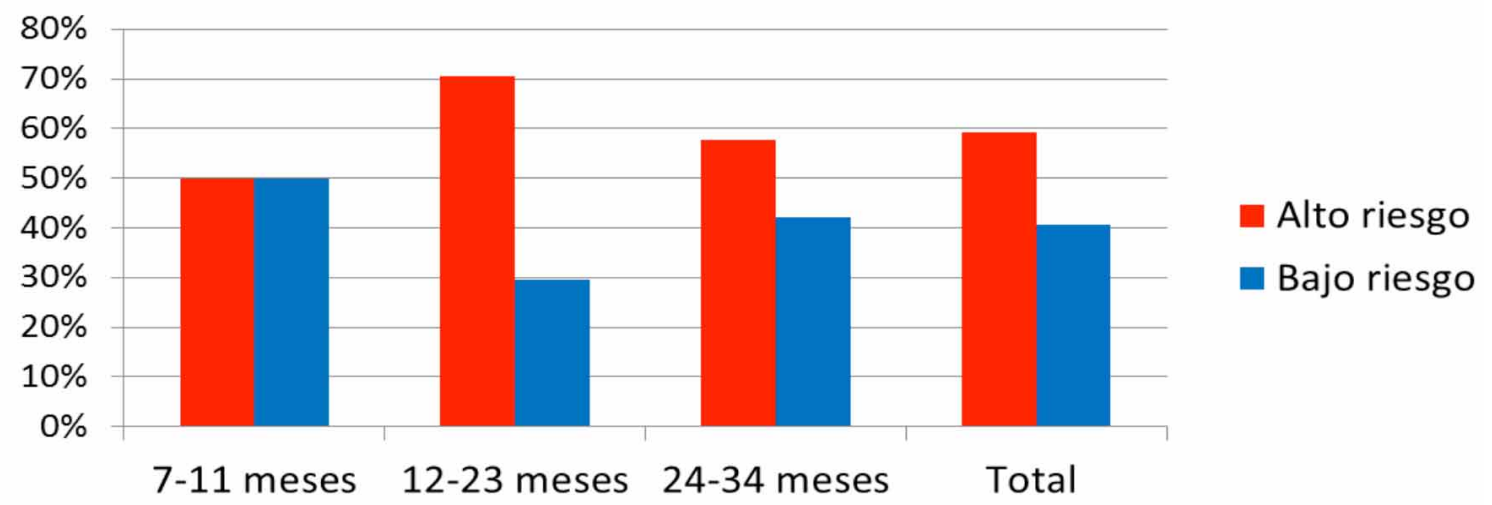

Fig. 2. Riesgo cariogénico por rango etario: En la población de 7-34 meses el 59 \% presenta riesgo alto. El segmento de 12-23 meses presenta el mayor riesgo con un $70 \%$, seguido de los segmentos de 24-34 meses y $7-11$ meses, con un $58 \%$ y $50 \%$, respectivamente.

Tabla I. Distribución del número de participantes por rango etario y sexo.

\begin{tabular}{lcccccc}
\hline Edad & Número & Porcentaje & $\mathrm{N}^{\circ}$ hombres & $\mathrm{N}^{\circ}$ mujeres & $\%$ hombres & $\%$ mujeres \\
\hline $7-11$ meses & 46 & $34 \%$ & 22 & 24 & $48 \%$ & $52 \%$ \\
$12-23$ meses & 44 & $33 \%$ & 22 & 22 & $50 \%$ & $50 \%$ \\
$24-34$ meses & 45 & $33 \%$ & 27 & 18 & $60 \%$ & $40 \%$ \\
Total & 135 & $100 \%$ & 71 & 64 & $53 \%$ & $47 \%$ \\
\hline
\end{tabular}


NENEN, A.; COURDURIER, C. \& ARCOS, A. Riesgo de caries en niños que ingresan al programa de Población en Control con Enfoque de Riesgo Odontológico. Int. J. Odontostomat., 13(4):437-441, 2019.

En el grupo 24-34 meses, los factores que presentaron mayor frecuencia de alto riesgo fueron: "uso de pasta con flúor", "momento de ingesta de azucares" y "cepillado antes de dormir", con un $71 \%, 67 \%$ y 44 $\%$, respectivamente.

El análisis global de los factores estudiados en toda la muestra (7-34 meses), arroja al "cepillado antes de dormir" como el factor con mayor porcentaje de alto riesgo, con un $60 \%$, seguido del "momento de ingesta de azucares" y "uso de pasta con flúor", con un $56 \%$ y $53 \%$, respectivamente (Fig. 3).

\section{DISCUSIÓN}

Los desafíos en salud deben ser enfrentados de tal modo que se continúe con las políticas que han sido exitosas, se modifiquen aquellas cuyos resultados no han sido los esperados y se innove en las metodologías y enfoques utilizados en los problemas de fondo y de larga data, que tienen repercusiones en el bienestar inmediato y futuro de las personas. En lo que respecta a la salud oral, las cifras de prevalencia de caries, sus características y distribución son conocidas (Ministerio de Salud, 2007). Además del estado y las secuelas que muestra la población (Ministerio de Salud, 2003; Arteaga et al.), por lo tanto, la existencia y desarrollo de programas curativos y rehabilitadores son indispensables. No obstante, la instauración de estas patologías se da a edades muy tempranas, con prevalencias de caries de $17,5 \%, 50,4 \%$ y $70,4 \%$, a los 2,4 y 6 años, respectivamente (Ministerio de Salud, 2007; Letelier et al.), cuya naturaleza es crónica y multifactorial (Selwitz et al., 2007). De este modo, se hace necesario el desarrollo de programas que prevengan la aparición de patologías como la caries, con estrategias adecuadas y en el momento más idóneo de la historia natural de la enfermedad y del ciclo vital de la persona. El programa de Población en Control con Enfoque de Riesgo Odontológico (CERO) es una iniciativa que cuenta con un enfoque y método eminentemente preventivo y de promoción de salud, tomando a la población infantil desde el inicio ( 6 meses) y controlando regularmente su estado de salud oraly los factores que influyen en la aparición de la enfermedad. La determinación del riesgo, la programación de controles basados en esta valoración individual, la búsqueda de la modificación de los factores de riesgo alterados a través de la implementación de medidas de prevención y promoción de la salud. Constituyen un cambio de paradigma en la atención primaria, llevándola a un enfoque centrado en la mantención del estado de salud, con el objetivo de aumentar la cantidad de población sana y buscando mantener dicha condición (Ministerio de Salud,

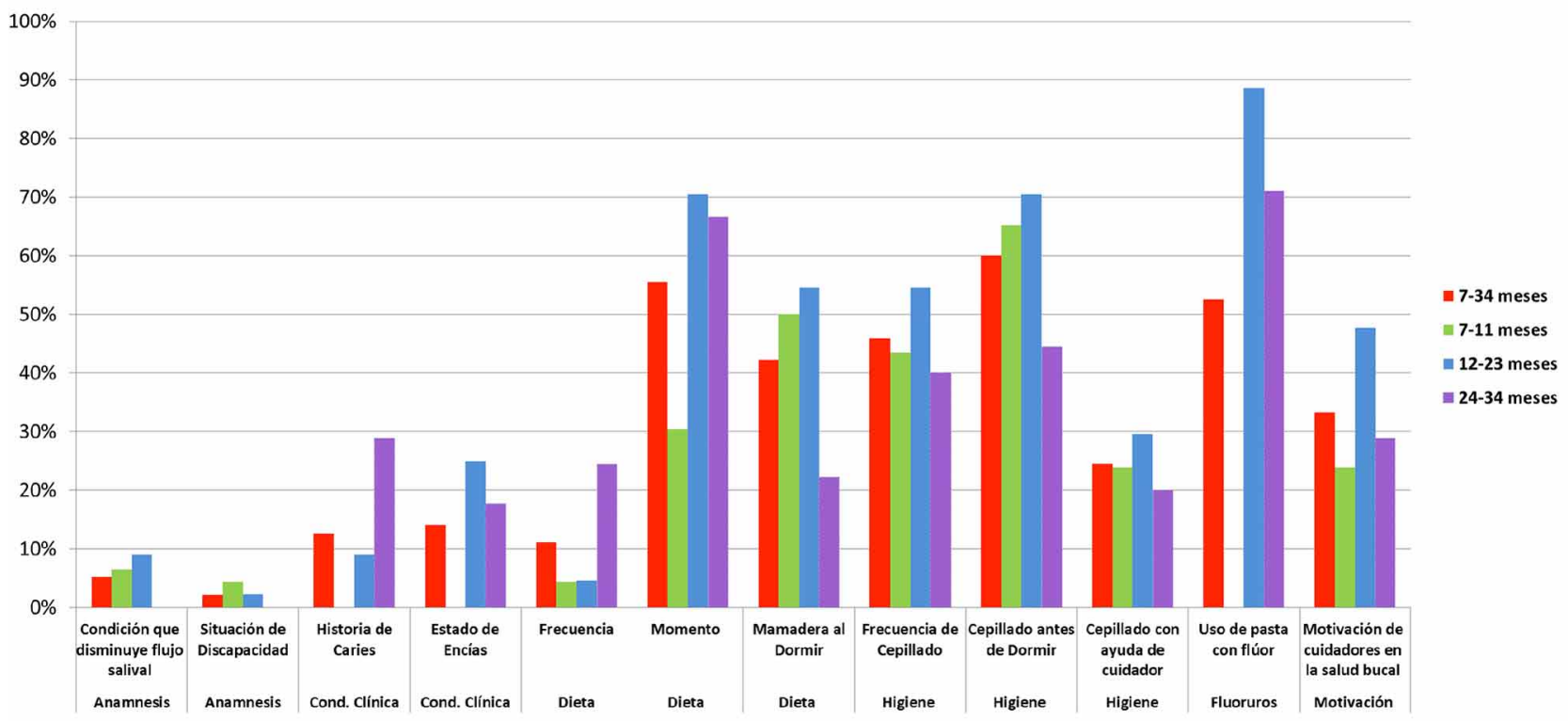

Fig. 3. Detalle de variables en alto riesgo: La variable de "cepillado antes de dormir" presentó el mayor porcentaje de alto riesgo en todo el grupo, con un $60 \%$. En el segmento de 7-11 meses la variable con mayor porcentaje en alto riesgo fue "cepillado antes de dormir" (65 \%) y en los segmentos de 12-23 y 24-34 meses, fueron "uso de pasta con flúor" con un $89 \%$ y $71 \%$, respectivamente. 
2017). Los resultados observados en esta investigación parecen tener relación con las cifras expuestas por estudios previos que evaluaron prevalencia de caries de niños en etapa preescolar y escolar (Ministerio de Salud, 2007; Letelier et al.), aportando una imagen completa y complementaria a la información epidemiológica existente, entregando datos reales y concretos del riesgo cariogénico que presenta la población infantil menor de 3 años. Estos resultados deben ser contrastados e integrados a otros provenientes de la implementación de este programa en otros centros de salud a nivel nacional. Logrando así, en etapas y análisis posteriores, un conocimiento y comprensión cabal del escenario existente en relación a salud oraly caries en la población. Dando herramientas relevantes para el correcto manejo e implementación de estrategias de control de estos factores de riesgo y el posible enlace de las medidas preventivas y de promoción entre los diferentes programas de salud existentes.

AGRADECIMIENTOS. Al Centro de Salud Familiar Quinto Centenario de la Ciudad de Osorno y a todo el equipo que trabaja en su unidad dental, por permitir y guiar este trabajo. A Natalia Mayorga, Angélica Solís, Francisca Coronado, Katerine Rojas y Erika Guzmán, por colaborar en la implementación del programa. A los padres, cuidadores y pacientes, por participar de este estudio.

NENEN, A.; COURDURIER, C. \& ARCOS, A. Dental caries risk in children who enter the program population in control with dental risk approach. Int. J. Odontostomat., 13(4):437-441, 2019.

ABSTRACT: The aim of this study was to determine the dental caries risk in the child population between 7 and 34 months, users of a primary health care center in the city of Osorno, Chile, admitted to the Population in Control with Dental Risk Approach program during the year 2017. The risk was expressed at high or low. A descriptive observational study was carried out, taking a sample of 135 patients, with ages within the range under study, enrolled in the health center and entered in the program. The guidelines designed by the Ministry of Health and included in the Population in Control with Dental Risk Approach program were used, applying them in a session, along with the clinical examination to determine the level of risk of each individual. Subsequently, the data were subjected to descriptive statistics analysis. $59 \%$ of the population between 7-34 months has a high dental caries risk. The group with the highest risk is $12-23$ months of age with $70 \%$. Men have a $59 \%$ high risk rate, and women 58 $\%$. When analyzing each variable in detail, the "brushing before sleep" was the most frequently found at high risk, with $60 \%$, followed by the "moment of intake of sugary foods" and the "use of paste with fluoride", with $56 \%$ and $53 \%$, respectively.

KEY WORDS: dental caries, risk factors, child health, public health, dental care.

\section{REFERENCIAS BIBLIOGRÁFICAS}

Arteaga, O.; Urzúa, I.; Espinoza, I.; Muñoz, A. \& Mendoza, C. Prevalencia de caries y pérdida de dientes en población de 65 a 74 años de Santiago, Chile. Rev. Clin. Periodoncia Implantol. Rehabil. Oral, 2(3):161-6, 2009.

Giacaman, R. A.; Miranda Reyes, P. \& Bravo León, V. Caries risk assessment in Chilean adolescents and adults and its association with caries experience. Braz. Oral Res., 27(1):7-13, 2013.

Gómez, P. Determinación del Riesgo Cariogénico Utilizando el Cuadro de Riesgo de la Ficha Clínica de Odontopediatría y el Programa Computacional Cariogram. Universidad de Talca, 2008: Estudio Piloto. Tesis para Optar al Título de Cirujano Dentista. Talca, Universidad de Talca, 2008.

Hänsel Petersson, G.; Twetman, S. \& Bratthall, D. Evaluation of a computer program for caries risk assessment in schoolchildren. Caries Res., 36(5):327-40, 2002.

Letelier, M.; Mendoza, C. \& Del Valle, C. Informe Consolidado del Diagnóstico Nacional de Salud Bucal de los Niños y Niñas de 2 y 4 Años que Participan en la Educación Parvularia. Chile 20072010. Santiago de Chile, Ministerio de Salud, Gobierno de Chile, 2012. Disponible en: https://diprece.minsal.cl/wrdprss minsal/wpcontent/uploads/2015/05/Informe-consolidado-2-y-4-a \%C3 \%B1os.pdf

Ministerio de Salud (MINSAL). Diagnóstico Nacional de Salud Bucal de los Niños de 6 Años. Chile 2007. Santiago de Chile, Ministerio de Salud, Gobierno de Chile, 2007. Disponible en: https:// diprece.minsal.cl/wrdprss minsal/wp-content/uploads/2015/05/ Diagnostico-Nac-Salud-Bucal-ni \%C3 \%B1os-6-a \%C3 \%B1os Chile-2007.pdf

Ministerio de Salud (MINSAL). Guía Clínica. Atención Primaria Odontológica del Preescolar de 2 a 5 años. Santiago de Chile, Ministerio de Salud, Gobierno de Chile, 2009. Disponible en: https:/ / w w w. m i n s a I. c I/ portal/ u r I/ i t e m / a86d289427cb092be04001011e01193c.pdf

Ministerio de Salud (MINSAL). Orientación Técnico Administrativa Población en Control con Enfoque de Riesgo Odontológico. Programa CERO. Santiago de Chile, Subsecretaría de Redes Asistenciales, Ministerio de Salud, Gobierno de Chile, 2017. Disponible en: https://diprece.minsal.cl/wp-content/uploads/2019/02/ Orientacion-Tecnica-Programa-CERO-2019.pdf

Ministerio de Salud (MINSAL). Resultados I Encuesta de Salud, Chile 2003. Santiago de Chile, Ministerio de Salud, Gobierno de Chile, 2003. Disponible en: http://www.medicinadefamiliares.cl/Protocolos/encnacsalres.pdf

Selwitz, R. H.; Ismail, A. I. \& Pitts, N. B. Dental caries. Lancet, 369(9555):51-9. 2007.

Dirección para correspondencia:

Ariel Nenen

Instituto de Odontoestomatología

Facultad de Medicina

Universidad Austral de Chile

Valdivia

CHILE

Email: ariel.nenen09@gmail.com

Recibido : 23-08-2018

Aceptado: 12-06-2019 